\title{
Testing times ahead? An exploration of laboratory use in Primary Care
}

\author{
Sharon Louise Cadogan
}

Department of Epidemiology \& Public Health, UCC

"Blood will tell, but often it tells too much" (Don Marquis)

\section{Demand management}

Healthcare budgets worldwide are facing increasing pressure to reduce costs and improve efficiency, while maintaining quality. Laboratory testing has not escaped this pressure. A major component of healthcare budgets, the demand for testing, is increasing at a faster rate than medical activity. And, while laboratory testing is undoubtedly a valuable first step in any diagnosis, the sheer volume of testing may be conducive to waste. Laboratory services cost the Health Service Executive (HSE) approximately $€ 469$ million each year. That is over 10 times the budget of a small hospital for a whole year. In terms of volume, there are over 76 million tests performed annually in Irish laboratories. These tests are made up of both urgent and non-urgent tests and originate from various sources including A\&E, inpatient, outpatient and primary care, with approximately half from the latter. Laboratory testing is an integral part of day-to-day primary care practice, with approximately $30 \%$ of patient encounters resulting in a request.

While the proportion of the health budget spent on laboratory services may appear relatively low at $3-4 \%$, the significant costs incurred are in fact the additional downstream costs such as; the further outpatient visits and x-rays, as well as the costs to the patient of more journeys to appointments, days off work, sleepless nights worrying, or even the excessive searching of the Internet to research the myriad conditions that they may or may not have.

Why is demand management important? The practice of medicine is not easy. The correct diagnosis and best treatment plan is not always clear. Therefore, with every episode of care, the resources expended to ensure a timely and accurate diagnosis may be pivotal. That is because they influence all other decisions thereafter in the delivery of care. Hence, before requesting any laboratory test, physicians should consider the aim of the test and have a clear understanding of how the result will be interpreted and how the patient's management will be affected by the result. Some medical staff would suggest that if a test result won't change the management of a patient, it should not be done.

In a recent article for the New Yorker, Dr. Atul Gwande, who is a surgeon and public health researcher as well as a best-selling writer, made an interesting comparison between 


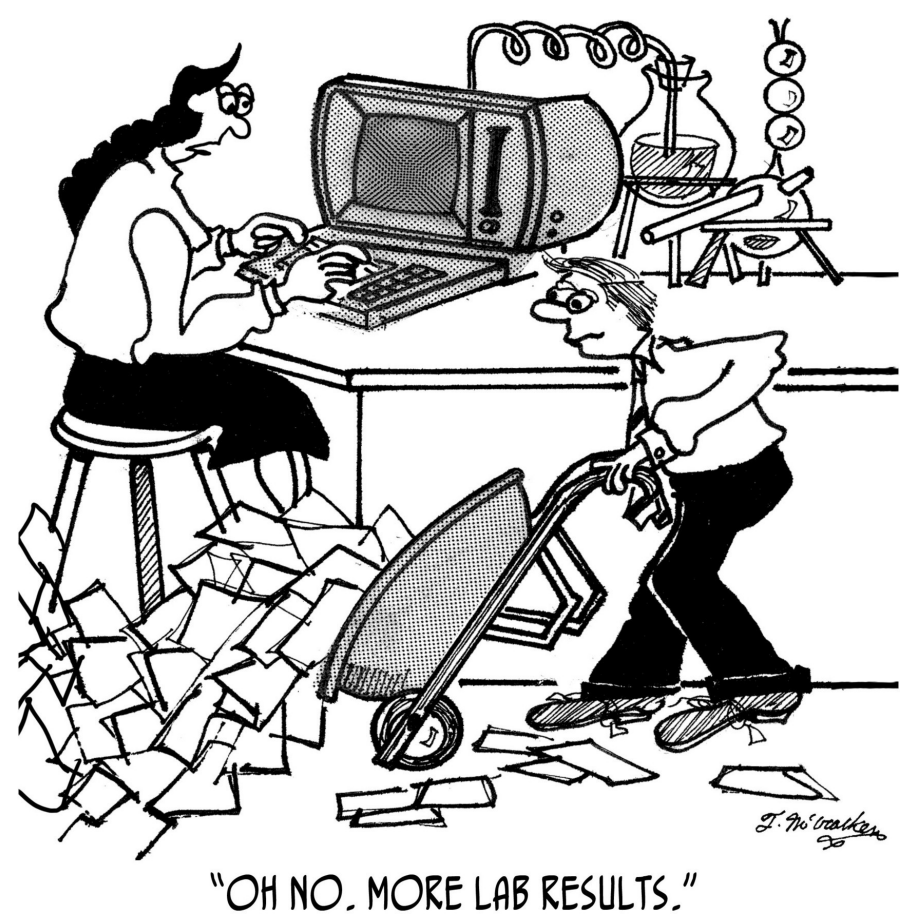

Figure 1: Oh no. More lab results. Source: www.cartoonstock.com

laboratory testing and fishing. He stated that "often, these are fishing expeditions, and since no one is perfectly normal you tend to find a lot of fish". Are we catching too many fish, and what will we do with them? With this in mind, the effective use of resources to perform the right test at the right time is essential for ensuring the quality of patient outcomes. Also, the value of any test is subject to how likely a patient is to have a significant problem in the first place. For example, tiredness or 'fatigue' is a symptom of many conditions including anaemia, coeliac disease, depression, diabetes, glandular fever and underactive thyroid, just to mention a few. However, a patient presenting with 'fatigue' may not need laboratory investigations, or at least not for each of these conditions. The decision to request a test needs to be considered in the wider context of the patient's clinical picture - an amalgam of their history and any findings on examination.

For example, a 31 year old male, married with two kids, who recently stepped onto the property ladder and started a new job, complains of 'chronic' tiredness. What are his chances of having underactive thyroid?

\section{It's about doing the right test on the right patient at the right time}

In my research, I noted that many General Practitioners (GPs) report having a 'battery of tests' that they perform in the first instance to rule out many conditions. Depending on the volume and type of tests involved, this may lead to another problem caused by overtesting - over-diagnosis. Now, many believe that the diagnosis of any medical condition 


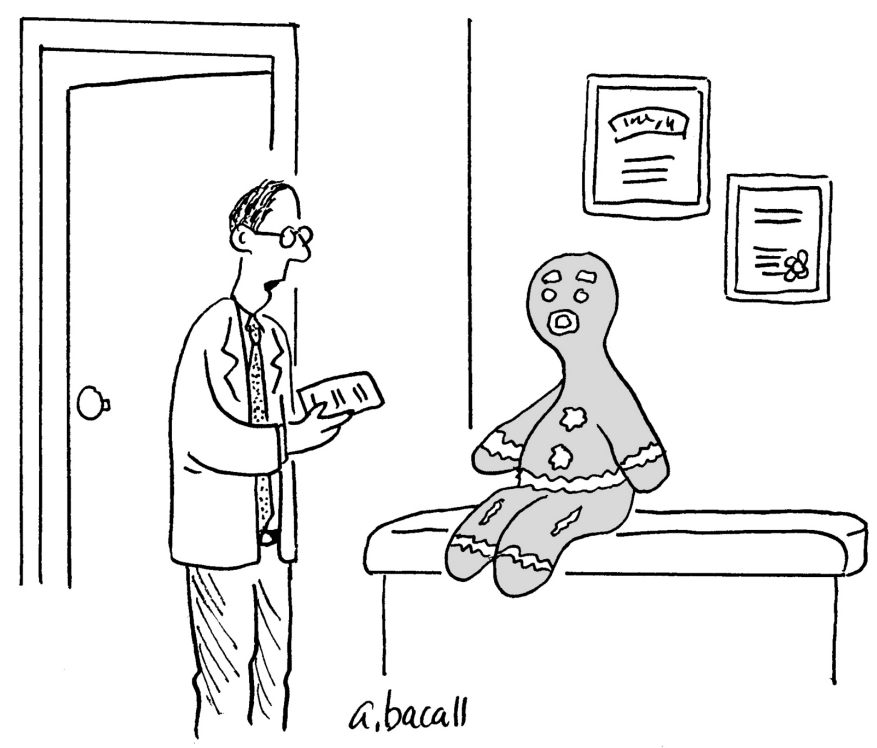

"I have your test results. Your sugar is too high."

Figure 2: I have your test results. Your sugar is too high. Source: www.cartoonstock.com

at an early stage is surely a good thing. And, of course in some cases it is, in particular for certain cancers where prognosis is dependent on early detection. However, in many other conditions, it may lead to the diagnosis of a condition that will never bother you in your lifetime. Imagine if you or a loved one were diagnosed with something that your doctor advises you to forget about because it will not harm you. Of course, you are not going to forget about it, you will begin thinking about the possibilities; how can you be sure it it harmless? Maybe I should have some treatment just in case?

Laboratory tests need to be used judiciously, and in particular should be mindful of the clinical context. The overuse, and similarly, underuse of laboratory services can stem from the physician, the patient and the broader policy context. For example, many physicians report uncertainty about when to order tests and how to interpret test results. Reasons given for this include lack of knowledge about indications, costs, insurance restrictions and inconsistent names for the same test. Similarly, patients play a role in the ineffective use of laboratory tests. Patients often have the perception that 'more care is better care' and, that extra testing may indicate a 'more thorough GP'.

Whatever the context or criteria, inappropriate testing can cause harm and lead to medical errors. Overuse can result in unnecessary blood tests and other procedures. Also, resolving the uncertainty of non-normal or borderline results may lead to further medical procedures that have costs of their own. It also increases the likelihood of false-positive results, which can lead to incorrect diagnoses, increased costs, and adverse outcomes due to unjustified additional intervention. Meanwhile, underutilization can result in morbidity due to delayed or missed diagnoses and in downstream overutilization. Overuse and 
underuse of tests can both lead to longer hospital stays and contribute to legal liability.

So why is this important? Literature suggests that as much as $70 \%$ of laboratory tests may not be needed. However, a laboratory test may be initiated an estimated $30 \%$ of patient encounters in primary care. Yet, no studies to date in Ireland have examined the use of laboratory tests in primary care.

\section{A case study: serum immunoglobulin tests}

It is likely that wasteful requesting of laboratory tests occurs across all tests, and similarly across all disciplines of medicine. However, it is not feasible to examine all tests, and, for the purpose of this research we decided to explore requests for quantitative serum immunoglobulins.

What are serum immunoglobulin tests and when should they be used? Serum immunoglobulin tests measure the level of immunoglobulins (antibodies), in the blood. Antibodies are proteins made by the immune system to defend against antigens, such as bacteria, viruses, and toxins. Quantitative serum immunoglobulin tests are used to detect abnormal levels. Low immunoglobulin levels characterise some antibody immunodeficiencies (when the immune system isn't working properly). These are really rare. Meanwhile, high immunoglobulin levels (polyclonal gammopathy) are often found in liver diseases, chronic inflammatory diseases, haematological disorders, cancers and infections.

\section{Aim of this research}

The aim of this PhD is to explore the efficiency of laboratory use among GPs in the CorkKerry region. This firstly involves identifying any GP variation in requesting patterns. This requires the extraction of data on all immunoglobulin tests requested by GPs in the Cork-Kerry region for a time period of one year.

So why does this physician-to-physician variation exist? That leads to the next component of this research; a qualitative study (using semi structured interviews) exploring GP attitudes on test requesting and referrals to outpatient clinics.

Both of these study components inform the remainder of the $\mathrm{PhD}$ : finding a plaster to control the excess blood. That is, an intervention to promote optimum laboratory use will be developed, implemented and evaluated for effectiveness. This intervention will be designed based on findings of previous two studies in the $\mathrm{PhD}$, along with the recommendations from the international literature on what has previously been effective.

\section{The impact of this research}

With testing times ahead for healthcare budgets, this research is timely and could have an important impact on reducing health service costs and laboratory workload, while 
Care

improving patient care. Regardless of the effectiveness of the intervention, this research highlights some key issues around testing, including uncertainty about when to test and difficulty interpreting the results.

It is anticipated that findings from this $\mathrm{PhD}$ will inform public policy and good practice guidelines for the use of laboratory test, making the testing times ahead, less testing!

I would like to thank my supervisory panel: Professor Mary Cahill, Professor John Browne and Professor Colin Bradley. I would also like to acknowledge the Health Research Board PhD Scholars Programme for funding this research. 